Caroline Arni

\section{Seelengesetze mit Gesellschaftswert. Weibliche Subjektwerdung und die Utopie menschlicher Perfektion in der feministisch- sexualreformerischen Liebesethik um 1900}

L'amour est à réinventer, on le sait. Arthur Rimbaud, 1873

1911 sieht der Historiker, Nationalökonom und Soziologe Robert Michels einen "Sonnenball " wam Horizont bereits auftauchen", wenn auch dessen "Strahlen [...] noch blass und matt sind und noch nicht die Kraft haben, neues Leben zu spenden" (Michels 1911, 55). Zwar mit gezügeltem Enthusiasmus, aber mit viel Gewissheit wird hier auf dem Gelände zwischenmenschlicher Intimität ein $u$ topos angesiedelt: Der Sonnenball nämlich, das ist die "neue Geschlechtsethik«, verstanden als "freiwillige Liebe« und "Mixtum compositum von gegenseitigem Verstehen, Kameradschaftsgefühl und Sinnlichkeit“ (Michels 1911, 18. u. 120). Und diese Verheißung einer neuen Form der Geschlechterbeziehung schimmert nicht aus einem Nicht-Ort jenseits des Gegebenen in dieses hinein, sondern leitet als Morgendämmerung eine erwartete Zukunft ein.

Dass Robert Michels' Entwurf einer neuen Geschlechtsethik als utopischer zugleich ein programmatischer war, entsprach ganz dem veränderten "spatio-temporalen Register" utopischen Denkens in der Moderne: Nicht mehr eigentlicher $u$-topos, wurde die Utopie nach 1800 zum Programm, das nach Realisierung "an jedem Ort" strebt und sich auf diese Weise als Handlungsorientierung in die Gegenwart einschreibt; sie erschien "nunmehr als nahe oder ferne Zukunft, auf welche die Menschheit unweigerlich zugeht" (Picon 2002, 25; Riot-Sarcey 1998). Dass umgekehrt dieser Entwurf als programmatischer zugleich ein utopischer war, hat nichts mit einem dem Liebesgefühl transhistorisch innewohnenden utopischen Überschuss zu tun. Vielmehr war es Ausdruck eines Zeitraums, in dem gesellschaftliche Verhältnisse wesentlich auch als Geschlechterverhältnisse neu geordnet wurden und diese Neuordnung im Sinn des Fortschrittsdenkens als eine progressive Vollendung menschlichen Potentials - als Verwirklichung der Utopie - gedacht war. Und in den Debatten über solche Neuordnungen wurde die heterosexuelle Liebe, in der Geschlechterverhältnisse als Geschlechterbeziehungen Form annehmen, mit entsprechender Bedeutung aufgeladen.

Die Grundzüge der Michels'schen Vision waren denn auch nicht originell; vielmehr'sind sie exemplarisch für eine Konzeption des heterosexuellen Paars, die um 1900 im Kontext sexualreformerisch-feministischen Denkens entworfen wurde: Im Kern steht dabei die auf Autonomie (freiwillige Liebe) und Egalität (Kameradschaft) gegründete Liebesbeziehung, die sinnliche und intellektuelle Intimität zusammen schließt, darin das ethische Potential der Menschheit realisiert und dadurch zu deren Vervollkommnung beiträgt. Dieses Konzept nährte sich aus Traditionen romantischer wie auch feministischer und utopisch-sozialistischer Liebesdiskurse des 19. Jahrhunderts und aus ihm sollte sich das nüchtern psychoökonomische Liebesverständnis der Beziehungsarbeit unserer Tage herausschälen. Zugleich aber muss es auch als historische Singularität begriffen werden, die ihre Voraussetzungen in einer spezifischen epistemologischen und politischen Zeitgenossenschaft hatte.

Drei zeitgleiche Diskurse sind dabei wesentlich: Erstens wurde dieses Liebeskonzept in Begriffen verschiedener Thematisierungszusammenhänge von den entstehenden Humanwissenschaften über die Sexualpolitik bis zum Feminismus formuliert; zweitens war es konfrontiert mit einem alle weltanschaulichen Entwürfe beherrschenden Krisendiskurs über den drohenden Zerfall der modernen Gesellschaft, und drittens wurde es aufgeladen mit der pathetischen Zukunftshoffnung auf eine zu höchstem Seelenadel revolutionierte und evolutionär perfektionierte Menschheit. Im Kontext dieser Konstellation liefen im hier behandelten Liebeskonzept zwei Dinge ineinander: feministische Forderungen nach Geschlechtergerechtigkeit sowie weiblicher Autonomie und Subjektwerdung einerseits und utopisch-programmatische Visionen einer vollendeten Menschheit andererseits, die für totalitär-eugenische Verwerfungen gedankliche Grundlagen lieferten. Von diesem Zusammenfallen handeln die folgenden Ausfuihrungen, in denen ich zeigen möchte, dass diese beiden Elemente in der logischen Pointe der neuen Geschlechtsethik nicht einfach qua Zeitgenossenschaft gleichzeitig anwesend waren.

Dabei interessiert hier weniger die in der Forschung bisher behandelte Frage, in welchem Ausmaß feministische Sexualreformerinnen eugenisch ausgerichtet waren und welche Motive oder Strategien dem zugrunde lagen. ${ }^{1}$ Zwar schließe ich an die These an, dass die Postulate einer "neuen" Geschlechter- und Sexualmoral dort zumindest stellenweise in eine "sozialdarwinistisch-eugenische Programmatik" mündeten, wo sie mit "Konzepten einer gesamtgesellschaftlichen Reform und Regeneration« verknüpft wurden (Frevert 2000, 171; vgl. hierzu auch Herlitzius 1995, 327 sowie Bublitz/Hanke/Seier 2000, 287-315). Wesentlich für meine Ausfuihrungen ist weiter die Beobachtung, dass feministisch-se-

1. Zum Stellenwert der Eugenik im sexualreformerisch ausgerichteten, so genannten radikalen Flügel der deutschsprachigen Frauenbewegung um 1900 existiert einige Literatur; vgl. u.a. ausführlich und differenziert sowie mit Akzent auf Helene Stöcker: Herlitzius 1995; u.a. austuhrlich und differenziert sowie mit Akzent auf Helene Stocker. Herlitzius 1995 ;
maßgebend für eine Sichtweise, die nach dem systematischen Stellenwert eugenischen Denkens im feministischen Diskurs der Jahrhundertwende fragt: Allen 1991 und 2000; jüngst Denkens im feministischen Diskurs der Jahrhundertwende fragt: Allen 1991 und 2000; jungst
die verschiedenen Interpretationen zusammenfassend Matysik 2008, 76-78. Vgl. generell zur die verschiedenen Interpretationen zusam
Eugenik Weingart/Kroll/Bayertz 1992. 
xualreformerische Autorinnen und Aktivistinnen im weitesten Sinne eugenische Argumente nutzten, um die von Zeitgenossen postulierte Antithese zwischen der "Selbsterfüllung der Frauen" und dem "Allgemeinwohl" zu widerlegen (Allen 1991, 52). Doch möchte ich mich auf die in der Forschung noch wenig behandelte Thematisierung des Liebesgefühls (nur ansatzweise, aber wegweisend Herlitzius 1995, 142ff.) und damit auf ein argumentatives Detail konzentrieren. Anhand einzelner Schriften ausgewählter Protagonistinnen und vermittels einer diskursanalytisch informierten Interpretation versuche ich, die Argumentationslogik zu rekonstruieren, die feministische Postulate mit einer verhängnisvollen utopischen Vision verkoppelte. ${ }^{2}$

\section{Die Liebe als Wissensobjekt und Politikum: Konturen des Liebesdiskurses im Schattenwurf sozialer Ängste}

Anders als es ein sexualitätsgeschichtlicher Fokus nahe legt, war um 1900 im Feld der zwischengeschlechtlichen Intimität Sexualität nicht das exklusive mot d'ordre. Gerade so sehr war die Liebe Gegenstand intensiver Diskursivierung. So stößt eine bibliographische Recherche rasch auf eine Vielzahl von Schriften, die sie im Titel führen und ein Erklärungsversprechen abgeben: Ethnologie der Liebe (Mantegazza 1886), Psychologie der Liebe (Danville 1894), Kulturgeschichte der Liebe (Günther 1899), Physik der Liebe (de Gourmont 1903) - und nicht zu vergessen Sigmund Freuds Beiträge zur Psychologie des Liebeslebens $(1910,1912,1918)$ oder Georg Simmels Fragmente aus einer Philosophie der Liebe (1907).

Freilich: wer Liebe sagte, meinte um 1900 häufig Sexualität. So ist Rémy de Gourmonts Physik der Liebe - wie aus dem Untertitel ersichtlich wird - eine Abhandlung »über den sexuellen Instinkt». Gleichwohl macht es Sinn, analytisch zwischen Liebe und Sexualität zu unterscheiden, und sei es nur, um festzustellen, dass und wann die Begriffe synonym sind, oder um Gelenkstellen im Diskurs aufzuspüren, an denen das eine vom andern abzweigt - etwa indem die Liebe, wie in diesem Zeitraum üblich, als die "veredelte" Form des "Geschlechtstriebs" definiert wird (so z.B. bei Günther 1899, 5).

Im Vordergrund steht aber hier nicht eine Analyse des Verhältnisses von Sexualitäts- und Liebesdiskurs. Was stattdessen interessiert, ist die Thematisierungsan-

2 Aus einer diskursanalytischen Perspektive geht es mir um eine Rekonstruktion des in einem spezifischen historischen und epistemologischen Kontext Sagbaren, das seinerseits im Sinne einer
historischen Deutungsmusteranalyse auf konkrete, aber immer gedeutete Handlunosprobleme beziehen ist. Im Fall der hier zentralen Autorinnen sind das die Bemühungen um einebleme zu von Ehe und Scheidung sowie der Stellung lediger Mütter und Burer Kinder Ih behe Reform Beziige uur punktuell, weil ich legen möchte; ebenfalls bleibe ich hier ganz auf der Ebene der Diskursentationslogik legen möchte; ebenfalls bleibe ich hier ganz auf der Ebene der Diskurse, vgl. aber zur $V$ ielfalt der praktizierten heterosexuellen Paarbeziehung im Kontext dieser Diskurse sowie
rechtsgeschichtlicher Entwicklungen:Arni 2004 . strengung, die in den exemplarisch genannten Titeln zum Ausdruck kommt. Das ganze Feld junger humanwissenschaftlicher Disziplinen - Ethnologie, Psychologie, Kulturgeschichte, Soziologie - nahm sich unter solchen Titeln die Liebe als Gegenstand vor und unterwarf sie seinem Theoretisierungswillen. ${ }^{3}$ Damit ging der Liebesdiskurs aus der philosophisch und literarisch geprägten Liebesessayistik des 19. Jahrhunderts in Ansätze von humanwissenschaftlichen 'Liebeswissenschaften über, die parallel zur scientia sexualis entworfen wurden und nach den spezifischen Rationalitäten des Liebesgefühls fragten. So schrieb etwa der Arzt und Schriftsteller Max Nordau in seinen Paradoxa unter dem Kapitel "Zur Naturgeschichte der Liebe" gegen die »emotionellen Duseler beiderlei Geschlechts" an, die beim Thema sogleich und ausschließlich zur "poetischen Literatur" griffen, und plädierte stattdessen für "kühlen Ernst und wissenschaftliche Unvoreingenommenheit" (Nordau 1885, 273). Der Anthropologe Paolo Mantegazza wollte die Moral in Liebesdingen auf die Füße der »modernen Wissenschaft« stellen (Mantegazza 1886, 2), und der Soziologe Robert Michels versuchte sich in einer Vergleichenden Liebeswissenschaft der europäischen Länder (Michels 1911).

Das ausgeprägte Interesse dieser Liebeswissenschaften an Fragen der Monogamie und Polygamie überschnitt sich mit sexualpolitischen Forderungen nach einer Reform oder gar Abschaffung der auf Dauer und Exklusivität angelegten Ehe, wie sie in Kreisen der anarchistischen, künstlerischen und intellektuellen Bohème und unter dem Schlagwort der freien Liebe und freien Erotik propagiert und auch praktiziert wurde (vgl. u.a. Kuhn/Kohser-Spohn 2001). Solche Postulate wurden auch aus explizit feministischer Perspektive formuliert, galt doch das Lieben der Frauen als von der Kette oder dem Gefängnis der Ehe - wie es die französische Anarchistin Madeleine Vernet nannte - noch eingezäunter und domestizierter als dasjenige der Männer, das sich im Winkel der Doppelmoral gewisse Freiheiten herausnehmen konnte. So ist die "Befreiung« des "sexuellen Lebens» der Frau eines der Hauptanliegen in Vernets 1905 erstmals publizierter und in der Folge weit verbreiteter Schrift L'Amour libre.

Seitens der bürgerlichen Flügel der Frauenbewegung schlug solcher Radikalität massiver Widerstand entgegen. Helene Lange nannte die verschiedenen Spielarten freier Liebe eine gedanken- und gefühlsverwirrende "Hurra-Erotik“, Resultat eines modischen "geistigen Konsums", »ein wenig Nietzsche, ein wenig Darwin, ein wenig Sozialismus, etwas Ibsen, Wilde und Maeterlinck", bref: ein »erotischer Monismus, wie ihn zu allen Zeiten der künstlerische Individualismus

3 Es sollen hier nur drei Gründe dafür angedeutet werden: Erstens war die Frage nach dem "Wesen der heterosexuellen Liebe um 1900 insofern akut, als die Gesellschaften der Jahrhundertwende der heterosexuellen Liebe um 1900 insofern akut, als die Gesellschaften der Jahrhundertwende
sich als in ihren Ordnungen bedroht wahrnahmen und die Geschlechterbeziehung eine wesentliche Dimension sozialer Ordnung ist. Zweitens dürfte die von Luhmann in diesem Zeitraum angesiedelte Entdeckung der malten Inkompatibilitätu von Liebe und Ehe mals Problem in der Ehe" (Luhmann 1994, 191) ein Theoretisierungsbedürfnis ausgelöst haben, für das sich die humanwissenschaftlichen Diskurse anboten. Drittens befanden sich diese in Phasen der Formierung und beginnenden Konsolidierung als Disziplinen, und zu solchen Phasen gehört das Geltendmachen von Erklärungsansprüchen hinsichtlich spezifischer Gegenstände. 
verkündet hat, dem einzig an der Kultur der Persönlichkeit gelegen war und der die Liebe recht eigentlich aus der Gebundenheit an soziale Verpflichtungen lösen wollte, um ihre ganz ungehemmte Schwungkraft dem einzelnen zuteil werden zu lassen" (Lange 1909, 147-153). Solche Hurra-Erotik musste einer Helene Lange suspekt sein, verweigerte sie sich doch mutwillig dem, was ihr als die große Problematik der Zeit erschien: die Frage, wie das unbeständige Liebesgefühl der Individuen auf Dauer gestellt werden konnte, ohne die sozialer Zusammenhalt und soziale Ordnung nicht möglich schienen.

Die Semantik des Lange'schen Einspruchs macht deutlich, wie sehr Auseinandersetzungen über die Geschlechterbeziehung um 1900 eingefärbt waren von zeitgenössischen Ängsten vor Desintegration und Anomie. In einem vielfach verästelten und ausgesprochen breitflächigen Krisendiskurs wurde der drohende Zerfall sozialer Bande vorgestellt als das Verhängnis moderner Gesellschaften, die Arbeitsteilung, Spezialisierung und Individualisierung auf die Spitze trieben. ${ }^{4}$ Individualismus versus soziale Verpflichtung - entlang dieser Frontlinie verlief auch die Diskussion über die Liebe, umso mehr, als sie eng verknüpft war mit der Debatte um die Ehe, die eben, je nach Perspektive, als unzulässige Einhegung des individuellen Gefühlslebens oder aber als notwendige Garantie für die Dauerhaftigkeit sozialer Bindungen angesehen wurde.

Wer aus sexualreformerisch-feministischer Perspektive um 1900 eine Reform der heterosexuellen Paarbeziehung in den Michels'schen Begriffen einer neuen Geschlechtsethik der herkömmlichen Sexualmoral und Geschlechterordnung entgegenhielt, musste auf diese Ängste reagieren. Dabei war schon der Begriff Ethik insofern Programm, als er einerseits eine Orientierung von Lebensführung postulierte, andererseits aber eine Kritik moralischer Vorgaben sein wollte (Matysik 2008). Recht verstandene Liebe, so das entsprechende Argument, auf das ich im Folgenden näher eingehen möchte, ist einerseits mehr als hedonistische Lustbefriedigung und zugleich hat sie andererseits keine moralischen Konventionen nötig, weil sie selbst ein ethisches Prinzip ist, das über der Front zwischen Individualismus und sozialer Verpflichtung steht: Sie wahrt beides und hebt den Gegensatz in sich auf, indem sie die ,Qualitäts von Individuen und Kollektiv zugleich steigert. An just dieser argumentativen Stelle verfing sich dieses Liebeskonzept in den Abgründen utopischer Perfektionierungsverheißungen.

Fast idealtypisch und unter dem Schlagwort des erotischen Idealismus gefasst, finden sich die Elemente der fraglichen Interpretation in den in Europa weit verbreiteten und intensiv diskutierten Schriften der schwedischen Reformpädagogin Ellen Key, auf die ich im Folgenden eingehe. ${ }^{5}$ Ergänzend ziehe ich mit

4 Vgl. zum Krisenbegriff als "struktureller Signatur der Neuzeit" die mittlerweile klassischen Überlegungen von Koselleck (1982, 627); vgl. jüngst zur "Krise» als Gegenstand und Kategorie Überlegungen von Koselleck (1982, 627); vgl. jün

5 Ellen Key (1849-1926), Tochter eines schwedischen Großgrundbesitzers, eignete sich in Privatunterricht und autodidaktisch eine künstlerische und literarische Bildung an und war in Stockholm als Lehrerin und Dozentin tätig. Furore macht Keys 1900 erschienen der deutschen Sexualreformerin und Feministin Helene Stöcker die prominenteste Verfechterin der so genannten Neuen Ethik bei; unter diesem Etikett hatte das hier behandelte Liebeskonzept im deutschsprachigen Raum publizistischen und auch organisationellen Niederschlag gefunden. ${ }^{6}$ Interessant sind diese beiden Autorinnen gerade deshalb, weil sie ihr feministisches Liebeskonzept ins Postulat einer auf menschheitliche Perfektion zielenden Selektion münden ließen, sich zugleich aber von eugenischer Programmatik distanzierten. Gerade indem ihr Denken weder von eugenischem Gedankengut determiniert war, noch einfach qua Zeitgenossenschaft in dessen Nähe geriet, macht es die Bedeutung einer auf Vollkommenheit zielenden programmatisch-utopischen Fassung des Liebesgefuihls deutlich. Im Vergleich dieser beiden Autorinnen konturiert sich außerdem der genealogische Ort des feministisch-sexualreformerischen Liebeskonzepts im 20. Jahrhundert: Während Key in der optimistischen Stimmung ungebrochener utopischer Programmatik formuliert, klingt in Stöckers Variante eine Ernüchterung an, welche die pragmatische Zurücknahme utopischer Entwürfe im Verlauf des 20 . Jahrhunderts antönt.

\section{"Freiheit der Lieber: Eine neue Liebesethik}

1904 war Ellen Keys Essayband Über Liebe und Ehe in deutscher Übersetzung erschienen. Nur ein Jahr später folgte das Buch Liebe und Ethik, in dem sich die Autorin gegen die missverständliche Rezeption des vorangehenden Bandes verteidigte: Fälschlicherweise habe man ihre Überlegungen einerseits in die Nähe der "Freien Liebe«, andererseits in diejenige der "Rassenveredler» (Key 1905, 14) gerückt. Damit war Key ins Kreuzfeuer einer besonderen Ausprägung der Front zwischen Individualismus und sozialer Verpflichtung geraten: hier die Verfechter hedonistischer Libertinage, die keinen dem individuellen Lustempfinden übergeordneten Wert anerkennen mochten, da die Befürworter einer rationalisierten Fortpflanzung, die das individuelle Fühlen dem Kollektiv unterordnen wollten. Die diesbezüglichen Klarstellungen Keys sind äußerst aussagekräftig; ich werde zunächst anhand ihrer Kritik an der Freien Liebe die Grundzüge ihres Liebes-

individualpädagogische und sozialreformerische Studie Das Jahrhundert des Kindes (1902 in deutscher Übersetzung); in der Folge nahm sie in 44 Büchern und 149 Zeitschriftenartikeln Stellung zur Kindererziehung und zur Frauenfrage. Vgl. Mann 2004.

6 Helene Stöcker (1869-1943) absolvierte ein Lehrerinnenseminar, nahm in Berlin als Gasthörerin Helene Stöcker (1869-1943) absolvierte ein Lehrerinnenseminar, nahm in Berlin als Gasthöreri geisteswissenschaftiche und nationalokonomische Studien auf, arbeitete mit Wilhelm Dilthey zuintion ab. Seit den $1890 \mathrm{er}$ Jahren engagierte sich Stocker in der Friedens- und Frauenbewegung, publizierte vielfach, gründete einen Frauenstimmrechtsverband und den Bund für Mutterschutz und Sexualreform Nach der nationalsozialistischen Machtergreifung emigriente Stocker nach Zürich, von da aus nach we Sehweden, in die Sowjetunion und schließlich nach New York. Vgl. zu Stöcker besonders Wickert 1991; außerdem: von Bockel 1991; Hackett 1984; Schlüpmann 1984.Vgl. zur Neuen Ethik u.a Matysik 2008, 55-91; Reinert 2000; Allen 1999; Lichtblau 1993; Gilcher-Holtey 1992. 
konzepts konturieren, um in einem anschließenden Teil aus ihrer Distanzierung vom "einseitigen Züchtungsstandpunkt« (Key 1905, 22) dessen utopischen Gehalt und seine Implikationen herauszuarbeiten.

In ihren 1904 erschienen Essays, schreibt Key, habe sie keineswegs gefordert, dass »der einzelne [...] das höchstmögliche $\mathrm{Maß}$ von erotischem Glück« erhalten solle (Key 1905, 11f.). Tatsächlich hat es da geheißen: Nicht für die "freie Lieber" gelte es zu kämpfen, sondern für die »Freiheit der Liebe«; »denn während die erstere dahin gelangt ist, Freiheit für jedwede Liebe zu bedeuten, bedeutet die letztere nur Freiheit für ein Gefühl, das des Namens Liebe wert ist" (Key 1914 [1904], 142). Dieses Gefühl entsteht zwar aus dem wankelmütigen erotischen Impuls und bewahrt ihn in sich. Es geht aber in ihm nicht auf, sondern ist auf das Zusammenfallen von seelischer und sinnlicher Zuneigung angelegt. Solche Liebe ist nichts weniger als "Andacht vor der Persönlichkeit» des andern, und in dieser Andacht wird die Persönlichkeit der Liebenden selbst "edel" (Key 1905, 13, 26, 49). Ganz sinngemäß argumentiert Helene Stöcker, wenn sie eine "Verfeinerung der Lebens- und Liebeskunst" postuliert, die das Lieben einerseits "von aller Verachtung des Physischen" befreit, es aber andererseits "nicht bloß auf sinnlichem Wohlgefallen an dem Anderen« beruhen lässt. Solche Liebe bezieht auch Stöcker auf die Persönlichkeit des geliebten Wesens: sie ist Teil einer "Kultur der Persönlichkeit", die das Wesentliche nicht im "Staat" oder im "Jenseits", sondern "im Menschen " findet und deshalb von "hoher Bedeutung" ist für die "individuelle Entwicklung". Und in solcher Liebe verbinden sich "Mann und Weib" als "ebenbürtige Persönlichkeiten« (Stöcker 1912,7-11).

So verstandene Liebe geht vom sinnlichen Impuls aus und stellt das Flüchtige erotischer Anziehung auf Dauer, indem sie es einbindet in ein Engagement für die einzigartige Persönlichkeit des geliebten Menschen, das wiederum die Persönlichkeitsentwicklung der liebenden Person befördert. Überwunden wird damit das, was den Verfechterinnen einer neuen Liebesethik an der Freien Liebe fahrlässig schien: das Interesse an der Geschlechtlichkeit des andern. Für Stöcker und Key war solches gefährlich nah an einer hegemonialen Ontologie, welche sich die Frauen als nicht individualisierte Geschlechtswesen dachte. Gegen diese geschlechterpolarisierte Konzeption entwarfen sie eine an Persönlichkeit orientierte Liebe als Medium weiblicher Individuierung und Subjektwerdung. ${ }^{7}$ Darin ließ dieses Liebeskonzept auch die dualistischen Liebesphilosophien des 19. Jahrhunderts hinter sich (vgl. hierzu Fraisse 1997). Hatten diese die Liebe als ein Band verstanden, das aus männlichem Großmut und weiblicher Selbstaufgabe

7 Wobei Helene Stöcker das Postulat einer von Geschlechtszuschreibungen freien weiblichen Individualität radikaler vertrat als Ellen Key, die es mit dem Ideal der Frau als Gattin und Mutter verband; vgl. hierzu Stöckers kritische Key-Lektüre in Stöcker 1906, 150f. Vgl. übergreifend und grundlegend zur Personalisierung der Liebe in der Moderne: Luhmann 1994; vgl. zum weiblichen Kampf um Individualität um 1900 Bührmann 2004. gewoben war, so implizierte eine Verbindung von zwei Persönlichkeiten als solchen Ebenbürtigkeit und damit auch Egalität.

Im Kontext der zeitgenössischen sozialistischen und feministischen Kritik an der Institution Ehe als ein von monetären, familienpolitischen oder repressivmoralischen Interessen gesteuertes Mittel zum Zweck war ein zentrales tagespolitisches Element dieser Liebesethik die Forderung nach freier Liebeswahl (die sich auch auf verschiedene Partner in Serie oder gar gleichzeitig richten konnte) sowie nach freier Wahl der Beziehungsform (Ehe oder Konkubinat, auf Dauer angelegt oder kurzfristig, leichte Scheidung etc.). ${ }^{8}$ Ellen Key wie auch Helene Stöcker vertraten diese Forderungen in ihren Schriften. Und bei beiden waren sie eingefärbt von einer über den Tag hinausgehenden Finalität:Wird mit der Kategorie der ebenbürtigen Persönlichkeit eine feministische Motivation in das Liebeskonzept eingezogen, so erhält die Liebe mit der Veredelung, Kultivierung oder Entwicklung solcher Persönlichkeit einen auf die Vervollkommnung der Menschheit gerichteten utopischen Sinngehalt als eigentliche Zweckbestimmung. Die Persönlichkeitswerdung Ebenbürtiger in einem universalistisch auf alle Menschen gemünzten Sinn als eine eigentliche Menschwerdung der Menschheit - darauf zielt die Liebe, die Key und Stöcker meinen. ${ }^{9}$

\section{Veredelung der Gattung: Die Mission der Liebe}

Die an Persönlichkeit orientierte Liebe mit ihrem an die Frauen gerichteten Individuierungs- und Gleichheitsversprechen war entsprechend für Key wie Stöcker kein allein auf das Individuum bezogener Wert - sie konnte es nicht sein in einem Zeitraum, in dem die Furcht vor einem aller Verantwortung für das Kollektive entpflichteten Individualismus das Krisenempfinden der Zeitgenossen nährte. So propagierte Key die Liebe nicht als "Gegensatz zur Pflicht», sondern als »die erste und grösste Pflicht« des Menschen (Key 1905, 45). Liebe ist "nicht nur der Trieb, durch den das Menschengeschlecht neue Mitglieder erhält, sondern der Trieb, durch den das Menschengeschlecht immer mehr mit einander verbunden und veredelt werden wird, in dem die Kinder von den Eltern die große Macht ererben, zu lieben, die dann nach allen Richtungen auf das Ganze zu-

8 Im Prinzip wurde hier die Deinstitutionalisierung der Ehe, die sich imVerlauf des 20. Jahrhunderts Im Prinzip wurde hier die Deinstitutionalisierung der Ehe, die sich im verlauf des 20. Jahrhunderts gewissermaßen praktisch durchsetzen sollte (vgl. grundlegend dazu Tyrell 1988), als Modell
propagiert. Die daraus sich ergebende Frage der Regelung der Elternpllicht bewältigte Ellen Kropagiert. indem sie das Postulat nach Deinstitutionalisierung beziehungsweise Privatisierung der Geschlechterbeziehung mit der Forderung nach Sozialisierung der Kinderbetreuung koppelte, Geschlechterbezieh
vgl. Key 2005, 30.

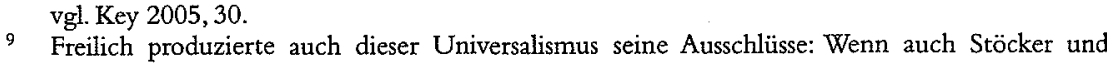
Key "die Menschheitu und gerade nicht nur eine bildungsbürgetliche Bohème in Sinn hatten so stand ihnen doch die zur Persönlichkeitsentfaltung freigesetzte Person ihres sozialen und kulturellen Milieus Modell. 
rückwirkt« (Key 1905, 37). Als Medium der Anerkennung und Entwicklung von Persönlichkeit ist die Liebe so zugleich teleologisch gerichtetes Medium sozialen Zusammenhalts (Verbindung) und menschlicher Perfektionierung (Veredelung). Das in der Liebe geadelte individuelle »erotische Glück» ist deshalb "zugleich ein Gesellschaftswert" (Key 1905, 69).

Solchen Gesellschaftswert aber - der Terminus des Ererbens deutet es an - hat die Liebe bei Key nicht nur im Sinn eines von jeder Generation neu zu erlangenden Seelenadels: Die "Hebung des Menschengeschlechts" im Medium der Liebe vollziehe sich "nicht nur wie früher durch die Selbstveredelung der Individuen in jeder neuen Generation, sondern auch durch den immer klarer sehenden Auswahlinstinkt der Individuen, für die Bedingungen, die darüber entscheiden, wie die Gattung fortgepflanzt wird" (Key 1905, 22). Die Evolution der Gattung mittels optimaler Fortpflanzung ist deshalb mitgemeint, wenn Key fordert, dass die "Gesellschaft" es "ermöglichen" müsse, "dass das erotische Glück der einzelnen der Veredelung der Gattung diene" (Key 1905, 12). Die recht verstandene Liebe ist in diesem Sinn zugleich Ziel und Mittel menschheitlicher Vollendung, da sie die transgenerationelle Weitergabe und allmähliche Verfestigung der Liebesfähigkeit garantiert.

Insofern als Key sich die personalisierte Liebe als Mechanismus evolutionärer Selektion denkt, der den Fortpflanzungstrieb gleichsam organisch in auf die Vollendung der Menschheit ausgerichtete Bahnen lenkt, weist sie die Postulate rationalisierter Reproduktion der "Rasseveredler" als verfehlt aus. Sähen jene nur in der "Elternschaft der am besten Ausgerüsteten - mit oder ohne Liebe, mit oder ohne Ehe - das für das Menschengeschlecht Bedeutungsvolle«, so sei umgekehrt "nur Elternschaft aus Liebe gut" (Key 1905, 14): »Aber dem einseitigen Züchtungsstandpunkt wurde in /Liebe und Eher die Hypothese entgegengehalten: dass die "Menschheit in der Liebe die die Gattung veredelndste Form der Auswahl gefunden hat" (Key 1905, 23). Gesteuerte Reproduktion müsse schon nur deshalb fehl gehen, weil "wir noch so wenig von den Bedingungen wissen, die die im physischen wie psychischen Sinne beste Nachkommenschaft versprechen (Key 1905, 28). Zwar, räumt Key im Wissen um die ja tatsächlich eben erst der Erforschung zugänglich gewordenen Gesetze der Vererbung ein (Rheinberger/ Müller-Wille 2009), herrschten auch im selektiven Mechanismus der Liebeswahl noch munentdeckte Gesetze" (Key 1905, 26). Dass aber das von zu strikten Eheund Scheidungsgesetzen befreite und von den Selektionsgewissheiten der "Rasseveredler" freie Liebesgefühl ein eigentlicher "Evolutionsfaktor" sei, der, wenn er in Freiheit wirken könne, auch die "Gesetze für eine allseitig lebenssteigernde Auslese« zu Tage treten lassen würde, das stand für Key fest (Key 1905, 24 u. 23).

Damit teilt sie, bei aller Kritik, mit den "Rasseveredlern« das Ziel optimaler Selektion - und nicht nur das: Auch bei ihr verläuft diese entlang von rassistischen Klassifikationen. So deutlich steht das zwar in der Verteidigungsschrift von 1905 nicht mehr, wohl aber in der Essaysammlung von 1904: Obschon die Liebes- wahl gegenwärtig "noch oft teils diesen Instinkt vermissen lässt, teils ihn kränkt«, falle sie doch "in Bezug auf nahe Blutsverwandtschaft, verschiedene Rassen und gewisse Krankheiten" bereits jetzt weitgehend mit ihm zusammen - so hindere etwa "kein Verbot, nur alle Impulse des Blutes [...] die Amerikanerin, einen $\mathrm{Ne}$ ger oder Chinesen zu heiraten“ (Key 1914 [1904], 157). ${ }^{10}$ Auch Helene Stöcker argumentiert in Übereinstimmung mit den "Rasseveredlern", wenn sie vor der Fortpflanzung »unheilbar Kranker und Entarteter» warnt (Stöcker 1905, 9).

Mit solchen Selektionsgewissheiten verwickeln sich beide Autorinnen in eklatante Widersprüche zu ihrem Postulat des personalisierten Liebesinstinkts, der doch auf nichts als die Persönlichkeit des andern achtet. In schlichter Widersprüchlichkeit aber geht diese Argumentation nicht auf, vielmehr stellt sie letztlich eine Spielart der Naturalisierung diskriminierender gesellschaftlicher Ordnungskategorien dar. Bei Key ist diese Naturalisierung insofern konsequenter noch als diejenige der "Rasseveredler», als sie ganz auf die Evolution des Instinkts vertraut und sich von "Zucht" - gewissermaßen als Kulturtechnik - distanziert Auch Stöcker lehnt staatsinterventionistische Zwangsmaßnahmen ab, wobei bei ihr stärker als bei Key die individuelle Freiheit maßgebend war: In ihrer liberalneomalthusianischen Perspektive waren auch eugenische Bestrebungen in Form von Pro- oder Antinatalismus Sache der individuellen Selbstverantwortlichkeit und allenfalls der sozialpolitischen Schaffung von Rahmenbedingungen; eugenische Argumente verknüpfte sie entsprechend mit Liberalisierungsforderungen hinsichtlich von Verhütung und Abtreibung und nicht mit Reglementierungsvorstellungen (vgl. Herlitzius 1995, 336-341; vgl. auch Wickert 1991, 67f. u. 80; zu den Tücken auch dieser Argumentation vgl. Allen 2000, 487).

Im Verhältnis zwischen dem feministisch-sexualreformerischen Liebeskonzept und zeitgenössischen eugenischen Postulaten hat die besonders deutlich von Key formulierte, nicht nur ideell, sondern auch biologisch evolutionäre Funktion der Liebe eine ambivalente Bedeutung: Bekräftigte sie, die als Einheit von Seelenund Gattungsadel quasi-natürlich auf eine Vervollkommnung der Menschheit angelegt ist, eugenische Postulate, so machte sie zugleich eine eugenische Programmatik recht eigentlich überflüssig, übernahm aber deren Kategorien der Selektion. Es war der in evolutionäre Theorien gekleidete utopische Überschuss, das Ausstatten einer Liebestheorie mit derVerheißung einer vollendeten Menschheit und nicht der simple Strom der Zeitgenossenschaft, der das feministisch-sexualreformerische Liebeskonzept ins Flussbett eines Denkens leitete, das in die totalitären Abgründe der Schaffung des Neuen Menschen münden sollte. Und da-

10 Dabei geht Key davon aus, dass sich kulturelle und biologische Evolution im Fortgang der Geschichte gleichsam organisch verbunden in dieselbe Richtung bewegen: "Die Liebesauswah ist schon in gewissen Fällen - wie in Bezug auf nahe Blutsverwandtschaft, verschiedene Rassen und gewisse Krankheiten - Instinkt geworden, nachdem Gesetz und Sitte die Auswahl so lange beeinflusst haben, dass diese ihrerseits Gefühl und Instinkt beeinflussen konnte." (Key 1914 [1904], 157) Damit übernimmt sie Postulate eugenischer und rassistischer Ehegesetze, indem sie sie gewissermaßen naturalisiert. 
bei war auch die im weitesten Sinn humanwissenschaftliche Thematisierung der Liebe nicht einfach zeitgleiche Erscheinung, sondern eigentliche Voraussetzung dafür, dass die Liebe überhaupt als ein Gegenstand der Suche nach ihr inhärenten Gesetzmäßigkeiten und Rationalitäten begriffen werden konnte.

\section{Schluss und Ausblick}

Im Kontext des seit dem 19. Jahrhundert formulierten Gegensatzes zwischen individueller Selbstbestimmung und gesellschaftlicher Ordnung, der um 1900 besonders in der Entgegensetzung von Ehe und Freier Liebe angesiedelt und beschworen wurde, bot sich das hier vorgestellte Liebeskonzept als vermittelnde Synthese an. Dabei ging es gerade nicht um eine "Ausgleichung nur zwischen den Forderungen der Gesellschaft an feste Eheformen einerseits und den Forderungen des einzelnen an Befriedigung des Sexuallebens in irgend einer Form andererseits". Im Sinn hatte dieses Liebeskonzept vielmehr "das neue Ausgleichungsproblem" zwischen der "Veredelung der Gattung auf der einen Seite und den gesteigerten Forderungen der einzelnen an Liebesglück auf der anderen Seite«. Eine Liebe, die zwischen solchen kollektiven und individuellen Ansprüchen vermittelt, ist mehr als Synthese: sie verspricht überdies, „lebenssteigernd" "für die Gattung wie für die einzelnen« zu sein (Key 1905, 21f.).

Via diese gedoppelte Verheißung verkoppelten sich feministische Forderungen mit selektionistischen Perfektionierungsutopien. Was rückblickend als eine anhaltend irritierende Verbindung erscheinen mag, entstand, indem die beiden Elemente voneinander abhängig gemacht wurden: Ist die Liebe Garantin einer lebenssteigernden optimalen Selektion hinsichtlich einer vollendeten Menschheit, so stützt umgekehrt das Interesse an solcher Perfektionierung die Forderung nach der Freiheit einer Liebe, in der das feministische Interesse an einer an Persönlichkeit und Ebenbürtigkeit orientierten und so das individuelle Leben steigernden Liebeskultur aufbewahrt ist. So mündete das 1873 zukunftsoffene L'amour est dे réinventer eines Rimbaud in den ersten Jahrzehnten des 20 . Jahrhunderts in die geschlossene Zukunftsvision einer biologisch und seelisch veredelten Gattung.

Der emotionale Aggregatszustand dieses Menschheitsadels allerdings fiel bei Ellen Key und Helene Stöcker sehr gegensätzlich aus: Rechnete die Pädagogin Key fest mit einem Endzustand vollkommener Harmonie, so setzte dem die in der Romantik bewanderte Stöcker einen Nietzscheanisch inspirierten Persönlichkeitskult entgegen, der die Erfüllung der Verheißung im Tragischen ansiedelt. $\mathrm{Ob}$ je eine perfekte "Harmonisierung" in Liebesdingen herrschen werde, argumentiert Stöcker in ihrem Key-Kommentar, scheine ihr nicht nur "zweifelhaft", sondern auch "unwesentlich«: „Denn nicht weichlicher Glücksgenuss ist das letzte
Ziel, sondern dass der Kampf und die Not des Lebens uns unbesiegt lassen, uns tiefer, freudiger, fruchtbarer machen. "(Stöcker 1906, 147) ${ }^{11}$

Das dominante Liebeskonzept der zweiten Hälfte des 20. Jahrhunderts sollte den Imperativ der Persönlichkeitswerdung aus dem Dienst menschheitlicher Veredelung herauslösen und ihm mit der im Fortgang der Geschichte diskreditierten Verheißung einer perfektionierten Menschheit die tragische Note einer Helene Stöcker ebenso nehmen wie das Harmonieversprechen einer Ellen Key. So ernüchtert wird er im psychologisch-ökonomischen Liebeskonzept unserer Tage zum Selbstzweck: Liebe ist hier immer schon als Beziehung gedacht, deren letzte Finalität die Entfaltung der Persönlichkeit der Liebenden ist - aber nicht im Durchgang durch die Tragödie à la Stöcker und nicht im Gefüge quasi-organischer seelengesetzlicher Harmonie à la Key, sondern nüchtern-pragmatisch im Sinne einer in täglicher Anstrengung zu leistenden Beziehungsarbeit.

DieVorstellung der Liebe als $u$-topos im eigentlichen Sinne hingegen sollte sich, allerdings ganz ohne gattungsveredelnde Einschläge, in gesellschaftstheoretischen Verhandlungen der Liebe halten:Von. Max Weber und Georg Simmel über Talcott Parsons bis zu Pierre Bourdieu dominiert in der soziologischen Theorie ein Verständnis der Liebe als Refugium nicht-rationalisierter und nicht-instrumenteller Beziehungen jenseits moderner Zerfallserscheinungen, in dem die Frauen mit ihrer naturwüchsigen Abneigung gegen moderne Vergesellschaftungszusammenhänge hausen. Um 1900 war auch diese Konzeption eine Antwort auf das Ausgleichungsproblem der Moderne - die allerdings anders als die feministischsexualreformerische Liebesethik nicht die Liebe, sondern die Geschlechterdifferenz theoretisiert. Beide Antworten mit ihren unterschiedlichen Implikationen für die Frauen gehören zu einer Genealogie der heutigen Ideale, Formen und Praktiken der Geschlechterbeziehung.

\section{Literaturverzeichnis}

Allen, Ann Taylor (2000): Feminism and Eugenics in Germany and Britain, 1900-1940: A Comparative Perspective. In: German Studies Review, Jg. 23, H. 3, S. 477-505.

Allen, Ann Taylor (1991): Feminismus und Eugenik im historischen Kontext. In: Feministische Studien, Jg. 9, H. 1, S. 46-68.

Allen Ann Taylor (1999): Feminism, Social Science, and the Meanings of Modernity: The Debate on the Origin of the Family in Europe and the United States, 1860-1914. In: American Historical Review, vol. 104, S. 1085-1113.

Arni, Caroline (2004): Entzweiungen. Die Krise der Ehe um 1900. Köln.

11 In dieser Differenz machte Stöcker selbst "den entscheidendsten Gegensatz" zwischen den Anschauungen Ellen Keys und ihren eigenen aus und führte ihn auch auf einen übersteigerten Idealismus zurück: Keys Ausführungen schienen oft "nur aus der reinen Idee geboren, als habe sie [Key] der harten, nüchternen Erfahrung nicht erlaubt, korrigierend einzugreifen«. (Stöcker 1906, Zitate auf S. 147 u. 149f.) 
Bublitz, Hannelore/Hanke, Christine/Seier, Andrea (2000): Der Gesellschaftskörper. Zur Neuordnung von Kultur und Geschlecht um 1900. Frankfurt a.M./New York.

Bührmann, Andrea (2004): Der Kampf um sweibliche Individualität". Zur Transformation moderner Subjektivierungsweisen in Deutschland um 1900. Münster.

Danville, Gaston (1894): Psychologie de l'amour. Paris.

De Gourmont, Rémy (1903): Physique de l'amour. Essai sur l'instinct sexuel. Paris.

Fraisse, Geneviève (1997): Von der sozialen Bestimmung zum individuellen Schicksal. In: Duby, Georges/Perrot, Michelle: Geschichte der Frauen, Bd. 4: 19. Jahrhundert (hg. von Geneviève Fraisse und Michelle Perrot). Frankfurt a.M., S. 63-95.

Freud, Sigmund (1999, zuerst 1908, 1912, 1918): Beiträge zur Psychologie des Liebeslebens. In: Ders.: Gesammelte Werke Bd.VIII Werke aus den Jahren 1909-1913. Frankfurt a.M.

Frevert, Ute (2000): Die Zukunft der Geschlechterordnung. Diagnosen und Erwartungen an der Jahrhundertwende. In: Dies. (Hg.): Das neue Jahrhundert. Europäische Zeitdiagnosen und Zukunftsentwürfe um 1900. Göttingen, S. 146-183.

Gilcher-Holtey, Ingrid (1992): Modelle "moderner" Weiblichkeit. Diskussionen im akademischen Milieu Heidelbergs um 1900. In: Rainer M. Lepsius (Hg.): Bildungsbürgertum im 19. Jahrhundert, Teil III: Lebensfuhrung und ständische Vergesellschaftung. Stuttgart, S. 176-205.

Günther, Reinhold (1899): Kulturgeschichte der Liebe. Ein Versuch. Berlin.

Hackett, Amy (1984): Helene Stöcker: Left-Wing Intellectual and Sex Reformer. In: Bridenthal, Renate/Grossmann, Atina/Kaplan, Marion (Hg.): When biology became destiny. Women in Weimar and Nazi Germany. New York, S. 109-130.

Herlitzius, Anette (1995): Frauenbefreiung und Rassenideologie. Rassenhygiene und Eugenik im politischen Programm der "Radikalen Frauenbewegung" (1900-1933). Wiesbaden.

Key, Ellen (1914, zuerst 1904): Über Liebe und Ehe. Essays. Berlin.

Key, Ellen (1905): Liebe und Ethik. Berlin.

Kuhn, Bärbel/Kohser-Spohn, Christiane (2001): Befreite Liebe. In: van Dülmen, Richard (Hg.): Entdeckung des Ich. Die Geschichte der Individualisierung vom Mittelalter bis zur Gegenwart. Köln/ Weimar/Wien, S. 489-516.

Koselleck, Reinhart (1982): Krise. In: Geschichtliche Grundbegriffe, Bd. 3. Stuttgart, S. 617- 650.

Lange, Helene (1996, zuerst 1909): Feministische Gedankenanarchie. In: Janssen-Jureit, Marielouise (Hg.): Frauen und Sexualmoral. Frankfurt a.M., S. 147-155.

Lichtblau, Klaus (1993):The Prostestant Ethic versus the »New Ethic». In: Lehmann, Hartmut/Roth, Günther: Weber's „Protestant Ethic». Origins, Evidence, Contexts. Cambridge UK, S. 179-193.

Luhmann, Niklas (1994): Liebe als Passion. Zur Codierung von Intimität. Frankfurt a.M.

Mann, Katja (2004): Ellen Key. Ein Leben über die Pädagogik hinaus. Frankfurt a.M.

Mantegazza, Paolo (1886): L'amour dans l'humanité. Essai d'une ethnologie d'amour. Paris (übers. aus dem Italienischen).

Matysik, Tracie (2008): Reforming the Moral Subject. Ethics and Sexuality in Central Europe, 1890-1930. Ithaca/London.

Michels, Robert (1911): Die Grenzen der Geschlechtsmoral. Prolegomena. Gedanken und Untersuchungen. München/Leipzig.

Nordau, Max (1885): Paradoxe. Leipzig.

Opitz-Belakhal, Claudia (2008): „Krise der Männlichkeit" - ein nützliches Konzept der Geschlechtergeschichte? In: L'Homme. EZFG, Jg. 19, H. 2, S. 31-49.

Picon, Antoine (2002): Les Saint-Simoniers. Raison, imaginaire et utopie. Paris.

Reinert, Kristen (2000): Frauen und Sexualreform 1897-1933. Herbholzheim.

Rheinberger Hans-Jörg/Müller-Wille, Stefan (2009): Vererbung: Geschichte und Kultur eines biologischen Konzepts. Frankfurt a.M.

Riot-Sarcey, Michèle (1998): Le réel de l'utopie. Essai sur le politique an XIXe siècle. Paris.
Schlüpmann, Heide (1984): Radikalisierung der Philosophie. Die Nietzsche-Rezeption und die sevulpolitische Publizistik Helene Stöckers. In: Feministische Studien, Jg. 3, H. 1, S. 10-38. Sexul, Georg (1985, zuerst 1907): Fragmente aus einer Philosophie der Liebe. In: Ders.: Schriften Simmel, Georg (1985, zuerst 1907): Fragmente aus einer Philosophie der Liebe
zur Philosophie und Soziologie der Geschlechter. Frankfurt a.M., S. 183-186.

Stöcker, Helene (1905): Zur Reform der sexuellen Ethik. In: Dies.: Mutterschutz, Jg. 1, H. 1, S. 3-12.

Stöcker, Helene (1906): Die Liebe und die Frauen, Minden.

Sök "Die neue Generation", 8.Jg., H. 3). Berlin.

Tyrell, Tuscher, Kurt/Schultheis, Franz/Wehrspaun, Michael (Hg.): Die "postmoderne« Familie. Familiale Strategien und Familienpolitik in einer Übergangszeit. Konstanz, S. 145-156.

Vernet, Madeleine (1905): L'amour libre. Paris.

Yon Bockel, Rolf (1991): Philosophin einer sneven Ethik :: Helene Stöcker (1869-1943). Hamburg. Won Bockel, Rolf (1911): Peter/Kroll, Jürgen/Bayertz, Kurt (1992): Rasse, Blut und Gene. Geschichte der Eugenik und Rassenhygiene in Deutschland. Frankfurt a.M.

Wickert, Christl (1991): Helene Stöcker (1869-1943). Frauenrechtlerin, Sexualreformerin und Pazifistin. Eine Biographie. Bonn. 